\title{
Hermann Ludwig Ferdinand von Helmholtz
}

Médecin, physiologue et physicien prussien, Hermann von Helmholtz est connu pour ses importantes contributions à l'étude de la perception des sons et des couleurs. On lui doit également d'avoir énoncé le principe de la conservation de l'énergie.

Riad HAIDAR, haidar@onera.fr

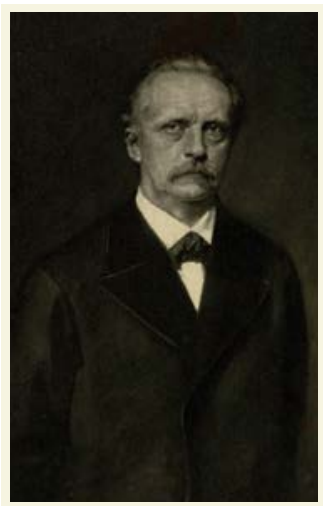

31 août 1821 Naissance à Potsdam (Prusse, actuelle Allemagne)

1847 Principe de conservation de l'énergie

1855 Théorie sur la perception des couleurs

1862 Travaux sur l'acoustique et la perception du son

1891 Création de la prestigieuse médaille Helmboltz

8 septembre 1894 Décès à BerlinCharlottenburg (Allemagne)

Portrait de Helmboltz par Franz von Lenbach en 1876.
H ermann von Helmholtz naît le 31 août 1821 dans une famille modeste de Postdam. Cette ville, qui héberge le palais de Sanssouci à quelques kilomètres de la capitale Berlin, sert alors de résidence à la maison royale de Prusse. À cette époque, c'est aussi une ville de garnison, au point que les trois quarts de sa population se composent de militaires.

Hermann est l'aîné d'une fratrie de quatre enfants. Sa mère Caroline, née Penn, est la fille d'un officier d'artillerie. Quant à son père, August Ferdinand Julius, il a servi dans les forces prussiennes et a combattu l'armée de Napoléon. Après la guerre, sa formation universitaire en philosophie lui a permis d'obtenir un poste d'enseignant au lycée de Postdam.

Hermann est inscrit au lycée de son père, et se passionne pour les cours de sciences physiques. Mais après l'Abitur, les finances familiales sont trop justes pour lui permettre de suivre des études supérieures. Le jeune homme brigue alors une des bourses que le gouvernement octroie au mérite, mais uniquement pour certaines filières triées sur le volet parmi lesquelles la médecine. Hermann renonce alors aux sciences physiques, et s'inscrit en fac de médecine à Berlin, à l'hôpital universitaire de la Charité qui est affilié à la prestigieuse Friedrich-Wilhelms-Universität (actuelle HumboldtUniversität zu Berlin).

Nous sommes en 1838, et il vient de quitter Postdam pour Berlin. En parallèle des cours de médecine, il a l'opportunité de suivre des cours de chimie et de physiologie. Et il étudie, seul, les mathématiques dans les livres de Laplace, Biot et Bernoulli. Petit à petit se forge dans son esprit la conviction d'un modèle mécano-chimique de la physiologie humaine, modèle qu'il défend dans son mémoire de doctorat.

\section{Premiers résulltats}

En 1843, tout juste diplômé, Helmholtz, qui doit 10 ans de service à l'état en compensation de sa bourse d'études, intègre un régiment militaire à Postdam.
Une fois les tâches quotidiennes expédiées, il passe son temps libre à mener des expériences. Il concentre ses efforts à démontrer que le mouvement musculaire repose sur une combinaison de mécanismes physiques et chimiques. En 1847, il publie ses idées dans Über die Erhaltung der Kraft où il investigue les principes mathématiques qui sous-tendent celui de la conservation de l'énergie. Il base ses réflexions sur les travaux de Carnot, Clapeyron et Joule... et y adopte d'emblée ce qui sera l'ossature de toutes ses futures publications : d'abord l'énoncé des arguments philosophiques, puis la description de la méthode et des résultats expérimentaux, et enfin le développement de l'argumentaire théorique pour identifier les lois générales.

Ce premier papier est véritablement novateur, et il établit immédiatement la renommée scientifique de Helmholtz. En investiguant la notion de travail, il y énonce en effet le principe de conservation de l'énergie cinétique, et l'applique à plusieurs situations expérimentales : il montre ainsi que, dans les situations où cette énergie semble perdue, elle est en fait convertie en chaleur. C'est aussi à cette époque qu'il entreprend l'étude, qui s'avérera extraordinairement féconde, des phénomènes sonores.

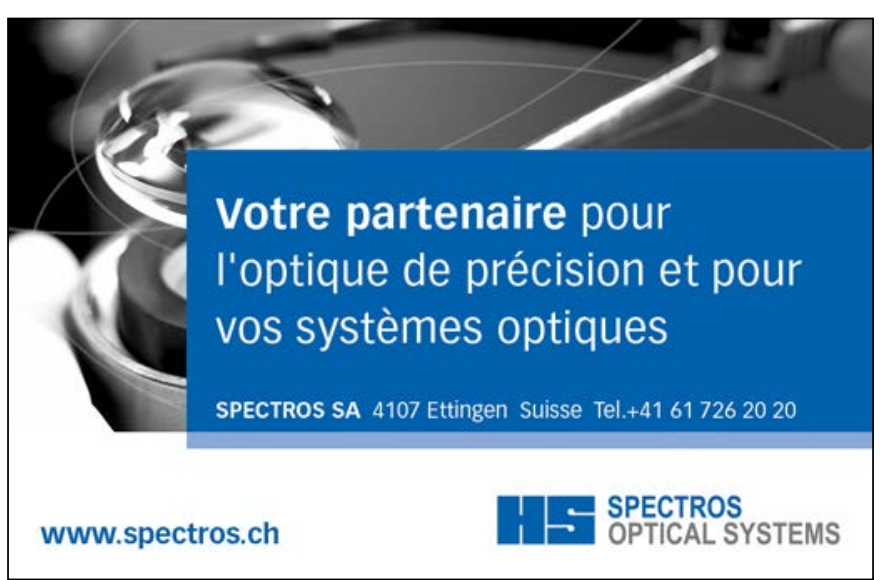


Fort de ces premiers succès, Helmholtz est relevé de ses obligations militaires bien avant l'échéance des 10 ans, ce qui lui permet de candidater (et d'obtenir) la chaire de physiologie à l'Albertus-Universität Königsberg en 1848.

L’année d'après, il épouse Olga von Velten et décide de s'établir durablement à Königsberg. Il s'engage ainsi dans une carrière académique, plus conforme à ses motivations profondes. Les premiers succès arrivent rapidement. Son invention de l'ophtalmoscope, notamment, lui vaut une reconnaissance immédiate et internationale. En 1852 et 1855, il publie des résultats majeurs sur l'optique physiologique en développant l'hypothèse initiée par Thomas Young, selon laquelle la perception de la couleur est due à la présence sur la rétine de trois types de récepteurs qui réagissent respectivement au rouge, au vert et au bleu. Cette théorie, vérifiée expérimentalement en 1859, porte désormais son nom (théorie de Young-Helmholtz).

\section{Pérégrimations à travers le royaume de Prusse}

Toutefois, sa situation à Königsberg se dégrade : il est conflit avec Franz Neumann, le professeur de physique, et surtout le climat froid de la ville ne convient pas à la santé fragile de son épouse. Il décide de déménager, et obtient la chaire d'anatomie et de physiologie à l'Universität Bonn en 1855. C'est à ce poste qu'il publie le premier volume de son Traité d'optique physiologique (Handbuch der physiologischen Optik) ainsi que, en 1858, un article fondateur sur le mouvement des fluides parfaits, dans lequel il établit sa loi sur les tourbillons. Cet article aura un impact considérable sur les travaux de Tait et Thomson, et il influencera toute l'école écossaise de physique mathématique.

Mais de nouveau, comme à Königsberg, sa situation devient inconfortable : en effet, certains esprits traditionnalistes n'apprécient pas son approche jugée trop mécanique de l'anatomie, et s'en plaignent auprès du ministère de l'éducation. Protégé par sa formidable notoriété, Helmholtz parvient à tenir bon, mais il n'en sort pas tout-à-fait indemne... Poussé (respectueusement, certes) vers la sortie, il finit par accepter la chaire d'anatomie à l'Universität Heidelberg, où on lui a promis la direction du tout nouvel Institut de Physiologie.

En 1862, il publie des travaux, également fondateurs, sur l'acoustique et la perception du son. S'inspirant des hypothèses initiales d'Ohm sur la notion de timbre, il formule une théorie mathématique de la résonance sonore qu'il appuie sur le puissant formalisme des séries de Fourier. Son inspiration et sa fécondité scientifiques sont alors à leur apogée mais, sur le plan personnel, il traverse une bourrasque émotionnelle : il perd en effet, coup sur coup, son père en 1858, puis son épouse Olga à la fin de l'année 1859. Âgé de 38 ans, veuf, avec deux jeunes enfants à charge, il décide de se remarier : il épouse Anna von Mohl, qui est la fille d'un collègue professeur à Heildelberg, en mai 1861. Anna, qui donnera trois autres enfants à Helmholtz, est une jeune femme attirante, rompue aux codes de la haute société, qui ouvre le foyer des Helmholtz et donne un nouvel élan à la vie sociale de son époux.

\section{Tourinant vers la physique}

À compter de 1866, dans la suite logique de ses travaux précédents, Helmholtz donne un tournant décisif à sa carrière. Il renonce aux problématiques classiques de la physiologie, et concentre désormais ses efforts sur les sciences physiques. Il s'intéresse notamment à l'électrodynamique, un thème sur lequel il a des échanges animés avec Weber tout au long des années 1870 - jusqu'au triomphe de Maxwell, et de son système d'équations.

Lorsqu'en 1870 la chaire de physique de la FriedrichWilhelms-Universität de Berlin devient vacante, il propose sa candidature. Kirchhoff est favori, car il a la renommée d'être un meilleur professeur, mais il décline l'offre que lui fait l'université. Helmholtz décroche alors le poste, assorti de l'engagement du gouvernement à construire un nouvel Institut de Physique. Cette fois, il a trouvé sa place. En 1877 et 1878, il assume même la charge de recteur de l'université. Son influence est considérable. Notons, en passant, qu'il accueille le jeune Heinrich Hertz dans son équipe de 1879 à $1883 .$.

\section{Hommeuirs et postérité}

Ses travaux lui valent de nombreuses reconnaissances: il est lauréat de la médaille Copley en 1873 et du Faraday Lectureship de la Royal Society of Chemistry en 1881; en 1883, il est élevé à la pairie ; en 1888, il devient le premier président de la Physikalisch-Technische Reichsanstalt. Enfin, honneur considérable, l'Académie des Sciences de Berlin décide de célébrer le $70^{\mathrm{e}}$ anniversaire de Helmholtz en créant, en 1891, la prestigieuse médaille Helmholtz qui récompense des universitaires qui ont accompli des avancées remarquables en sciences.

Helmholtz décède brusquement le 8 septembre 1894, à l'âge de 73 ans. Il repose au cimetière Wannsee de Berlin. Sa tombe, conçue par le sculpteur Adolf von Hildebrand, est consacrée depuis 1967 comme tombe honorifique de la ville de Berlin.

\section{POUR EN SAVOIR PLUS}

[1] J. J. O'Connor, E. F. Robertson, MacTutor History of Mathematics (2001)

[2] B. I. Tshisuaka, Hermann Ludwig Ferdinand von Helmholtz, Enzyklopädie Medizingeschichte (De Gruyter, Berlin/New York, 2005) 